Mancera G. (2020). Imaginando cómo podría ser el conocer reflexivo de los Bernabé Bernal. Revista Latinoamericana de Etnomatemática, 13(4), 50-69. DOI: 10.22267/relatem.20134.71

Artículo recibido el 17 de octubre de 2020. Aceptado para publicación el 26 de noviembre de 2020

\title{
Imaginando cómo podría ser el conocer reflexivo de los Bernabé Bernal
}

\section{Imagining how could it be the reflexive knowing of the Bernabé Bernal \\ ones}

Gabriel Mancera Ortiz ${ }^{1}$

\section{Resumen}

El presente artículo se configuró a partir de la versión del proyecto de investigación "Conocer reflexivo en contextos de modelación matemática desde una perspectiva socio crítica", centrando su atención, en tanto problema de investigación, en el cuestionamiento ¿Qué caracterizaría el conocer reflexivo de los Bernabé Bernal si en su historia escolar la modelación matemática desde la perspectiva socio crítica resultara ser un medio regular en su formación? Particularmente, se busca reportar el análisis de una experiencia de aula la cual se basa en un ambiente de modelación matemática, pensado desde la perspectiva socio crítica, en la que busca relacionar características del conocer reflexivo que desarrollan un grupo de estudiantes de grado undécimo en un colegio público de Bogotá - Colombia. Se inicia con una idea metodológica de investigación denominada investigación crítica, la cual pone en consideración que en una investigación podría tenerse en cuenta no solo lo que está ocurriendo sino también lo que podría haber ocurrido y lo que podría ser imaginado, los datos (registrados en grabaciones de audio y video, entrevistas, escritos y producciones de los estudiantes) fueron analizados, considerando la noción de los actos dialógicos. Luego se presenta al conocer reflexivo y la modelación matemática a partir de una perspectiva política de la Educación Matemática. De esta manera, se considera que el contexto en que los estudiantes desarrollan tal conocer es un elemento del punto de partida que debemos tener en cuenta en nuestras prácticas pedagógicas e investigativas. Así, se destacan varias reflexiones, entendidas como un abrebocas para iniciar la discusión en torno a cuatro rasgos distintivos del proceso desarrollado por los estudiantes: pensando en el otro, lectura crítica y matemática, prácticas con las matemáticas y la colectividad. Con este marco se caracterizan tales rasgos como características del conocer reflexivo desarrollado por los estudiantes.

Palabras clave: Modelación matemática; Conocer reflexivo; Imaginación pedagógica

\begin{abstract}
The present article is based on the version of the research project "Reflective knowing in mathematical modeling contexts from a social-critical perspective", focusing its attention, as a research problem, on the question: What would characterize the reflective knowledge of the Bernabé Bernal's if in their school history mathematical modeling from a social-critical perspective turned out to be a regular means in their training? In particular, this article seeks to report the analysis of a classroom experience based on a mathematical modeling environment, thought from a social-critical perspective, which sought to relate characteristics of reflective knowledge developed by a group of eleventh grade students in a public school in Bogota - Colombia. It starts

\footnotetext{
${ }^{1}$ Profesor de la Universidad Distrital Francisco José de Caldas, Bogotá, Colombia, Doctor en educación de la Universidade Federal de Minas Gerais, Belo Horizonte, Brasil, gmancera@yahoo.com / gmancerao@udistrital.edu.co.
} 
Mancera G. (2020). Imaginando cómo podría ser el conocer reflexivo de los Bernabé Bernal. Revista Latinoamericana de Etnomatemática, 13(4), 50-69. DOI: 10.22267/relatem.20134.71

with a methodological idea of research called critical investigation, which takes into consideration that in an investigation it could be taken into account not only what is happening but also what could have happened and what could be imagined. The data (recorded in audio and video recordings, interviews, students' writings and productions) were analyzed, considering the notion of dialogical acts. It is then presented to reflective knowledge and mathematical modelling from a political perspective of Mathematical Education. In this way, the context in which students develop such knowledge is considered an element of the starting point that we must take into account in our pedagogical and research practices. Thus, several reflections are highlighted, understood as an opening for discussion around four distinctive features of the process developed by students: thinking of the other, critical reading and Mathematics, practices with mathematics and the community. With this framework, such features are described as characteristics of the reflective knowledge

Key words: Mathematical Modeling; Reflective Knowing; Pedagogical Imagination

\section{Consideraciones iniciales}

En el presente artículo reporto el análisis de una experiencia de aula la cual se basa en un ambiente de modelación matemática, pensado desde la perspectiva socio crítica, en la que se buscó relacionar características del conocer reflexivo que desarrollan un grupo de estudiantes de grado undécimo en un colegio público de Bogotá - Colombia. Tal propósito, se configura a partir de un análisis al trabajo que presenté en Mancera (2020).

Para ello, parto por señalar que cuando era estudiante de educación básica, mi profesor de la asignatura de español nos propuso, como actividad académica, la lectura de la novela titulada Un tal Bernabé Bernal, del autor colombiano Álvaro Salom Becerra (1922 - 1987).

Esta novela narra las circunstancias de vida de Bernabé Bernal, un personaje que entre otros aspectos es configurado como: i) ingenuo, tímido e inteligente (aparentemente sin importancia) que trabaja como burócrata en una época de crisis política en la Bogotá del siglo pasado; ii) explotado por sus jefes y sus superiores para lograr sus aspiraciones sociales, políticas y económicas (aprovechándose, justamente, de esa ingenuidad, timidez e inteligencia); iii) culto y honesto pero cobarde, rebelde pero débil, idealista pero meticuloso; iv) que vive dentro de un círculo de escasez, de falta de oportunidades, de la lucha diaria por su sustento y el de su familia; v) con privaciones, anhelos, donde tal escenario le impidió, por ejemplo, culminar sus estudios, y a pesar de ello logró aprendizajes por sí mismo de manera reflexiva (autodidacta); y vi) que no traicionó, a pesar de sus circunstancias de vida, sus principios de moralidad y honradez, teniendo como gran satisfacción el deber cumplido y el orgullo de heredarles a sus hijos un nombre limpio y honrado. 
El fenómeno que Álvaro Salom Becerra ha narrado en este libro tiene conexiones con tantos otros libros que relatan y se constituyen en una denuncia sobre aspectos de la desigualdad y la vulnerabilidad social en Latinoamérica (no sólo en Colombia), las cuales han estado vigentes en la historia de América Latina.

Lo anterior, me ha llevado a pensar, apoyado en García (2002), en la posibilidad de modificar la articulación de investigaciones culturales, políticas y comunicacionales (y añado yo, curriculares), de lo contrario no podemos esperar que las voces y las imágenes de lo bogotano, colombiano, y en general de lo latinoamericano, sean otras que, por ejemplo, "las relatadas en el realismo mágico difundido por editoriales europeas y/o la de la descomposición social filmada en los noticieros de CNN o en las películas hollywoodenses" (p. 57); o las preestablecidas por currículos escolares que consideran a los estudiantes como meros sujetos cognitivos (como si fueran "caras"), desconociendo anhelos, sentires y porvenires (su rostro).

Por esta razón, en Mancera (2020) considero como posibilidad (en tanto ejercicio de la imaginación pedagógica) lo que podría sobrevenir con los Bernabé Bernal si los intereses académicos que ponemos en juego en nuestras propuestas curriculares también contemplan un fuerte componente social y político. Bajo estas ideas iniciales, en Mancera (2020, p. 204) planteo la pregunta: “QQué caracterizaría el conocer reflexivo de los Bernabé Bernal si en su historia escolar la modelación matemática desde la perspectiva socio crítica resultara ser un medio regular en su formación?”. Problemática que busco poner en discusión, como ya lo señalé, apoyado en algunas reflexiones establecidas en Mancera (2020).

Teniendo presente este fin, comienzo por ubicar la idea de la perspectiva socio crítica de la modelación matemática en la educación matemática ${ }^{2}$ como uno de los elementos de este ejercicio de imaginación pedagógica. Para ello, parto por señalar, como lo mencioné en Mancera (2020), que mi ingreso como profesor al proyecto curricular de la Licenciatura en Educación Básica con Énfasis en Matemáticas (LEBEM), hoy conocida como Licenciatura en Matemáticas (LEMA), de la Universidad Distrital Francisco José de Caldas (UDFJC), en Bogotá, Colombia, me permitió tomar consciencia que la resolución de problemas es una

\footnotetext{
${ }^{2}$ Para evitar repeticiones, a lo largo de este documento me referiré a la modelación matemática en la educación matemática como modelación, modelación matemática o bajo la sigla MM.
} 
Mancera G. (2020). Imaginando cómo podría ser el conocer reflexivo de los Bernabé Bernal. Revista Latinoamericana de Etnomatemática, 13(4), 50-69. DOI: 10.22267/relatem.20134.71

actividad matemática que podría posibilitar una toma de distancia de prácticas pedagógicas escolares predominantes, caracterizadas por un hacer repetitivo y sin sentido.

Bajo esta línea, la actividad de la modelación matemática, enunciada en documentos oficiales emanados por el Ministerio de Educación Nacional (MEN) como el de los lineamientos curriculares (Ministerio de Educación Nacional, 1998), dejó de ser para mí utópica (ideal) y sin sentido toda vez que, el actuar de los estudiantes de la LEBEM-LEMA al relacionarse con la resolución de problemas, interactúan con modelos matemáticos con los cuales en la mayoría de los casos buscan dar cuenta de la solución a la problemática planteada y de significar la actividad matemática misma.

En este sentido, encuentro hoy a la modelación matemática como una actividad importante que posibilita otra forma de pensar el trabajo en el aula de clase, en tanto es considerada como un método de enseñanza y de investigación, con el que se espera facilitarle al estudiante: i) integración de la matemática con otras áreas del conocimiento, ii) interés por la matemática frente a su aplicabilidad, iii) mejoría de la aprehensión de los conceptos matemáticos, iv) estímulo a la creatividad en la formulación y resolución de problemas, v) habilidad en el uso de máquinas (calculadora gráfica y computadoras), vi) capacidad para actuar en grupo, vii) orientación para la realización de la investigación, y viii) capacidad para la redacción de esa investigación (Hein \& Biembengut, 2006, p. 108).

Adentrándonos en la perspectiva socio crítica de la modelación, me apoyo en Barbosa (2006), quien propone concebir a la actividad de la modelación matemática como un terreno rico para la construcción de prácticas que tengan en cuenta, por una parte, el papel que desempeñan las matemáticas en la sociedad $\mathrm{y}$, por otra, la necesidad de apoyar el pensamiento crítico alrededor de dicho rol (prácticas socio críticas), concibiendo, de esta manera, una perspectiva crítica de la modelación matemática la cual resalta la reflexión tanto del papel de las matemáticas en la sociedad, como de la naturaleza crítica de los modelos matemáticos en la misma.

Para ello, como un hecho importante y coherente en tanto ejercicio académico, me apoyo en la idea de la Imaginación pedagógica (Skovsmose, 2015; Skovsmose \& Borba, 2004; Vithal, 2000). Por otra parte, centraré las reflexiones usando como técnica un ejercicio narrativo el 
cual no busca ser lineal; es decir, los apartados con los que daré fuerza a la reflexión pueden no seguir un patrón predeterminado, ello quedará a juicio del lector.

\section{Imaginación pedagógica: una relación para posibilitarnos otras miradas}

Aceptar que la realidad es moldeada por una serie de factores, que pueden ser entre otros: sociales, políticos, culturales, económicos, étnicos y de género, que se cristalizan en una serie de estructuras no es un asunto novedoso. Sin embargo, esta premisa implica, apoyado en Guba et al. (1994), considerar un paradigma de investigación en el que:

- La carga teórica y los hechos son interdependientes, pues los hechos solo lo son dentro de algún marco teórico.

- No se considera la subdeterminación de la teoría, pues los hechos no solo están determinados por la ventana teórica a través de la cual se buscan sino que, a su vez, distintas ventanas teóricas pueden estar igualmente sustentadas por una misma serie de hechos.

- Así como las teorías y los hechos no son independientes, tampoco lo son los valores y los hechos.

- Los hallazgos son creados por la interacción entre el investigador y el fenómeno, lo que me aleja, como ya he mencionado, de la idea de que los descubrimientos se dan a través de la observación objetiva de "cómo son realmente" los objetos y “cómo funcionan". (Mancera, 2020, p. 74)

Esta implicación, me ha posibilitado asumir que en una investigación, como ya lo señalé, se puede tener en cuenta no solo lo que está ocurriendo sino también, a partir de la idea imaginación pedagógica, lo que podría haber ocurrido y lo que podría ser imaginado, circunstancia que me permitió significar, además de considerar, la metodología de investigación denominada Investigación crítica.

Aceptar estas ideas resulta clave en mi camino de encontrar posibles respuestas a la pregunta ¿Qué caracterizaría el conocer reflexivo de los Bernabé Bernal si en su historia escolar la modelación matemática desde la perspectiva socio crítica resultara ser un medio regular en su formación? La cual podría no contestarse, probablemente, si no tenemos en cuenta la premisa "lo que no es y lo que podría ser construido".

En consonancia con lo anterior y siguiendo los planteamientos de Skovsmose (2015), Skovsmose \& Borba (2004) y Vithal (2000), la investigación crítica tiene en cuenta tres situaciones: actual (SA), imaginada (SI) y dispuesta (SD), y consecuentemente tres 
Mancera G. (2020). Imaginando cómo podría ser el conocer reflexivo de los Bernabé Bernal. Revista Latinoamericana de Etnomatemática, 13(4), 50-69. DOI: 10.22267/relatem.20134.71

relaciones: imaginación pedagógica (IP), organización práctica $(\mathrm{OP})$ y razonamiento crítico (RC).

Para estos autores, la situación actual contiene la mirada de lo que sucede y acontece en el contexto donde se lleva a cabo la investigación y en la que los sujetos que participan en la investigación van a cuestionar ese acontecer y van a problematizarlo.

Justamente tal problematización es la que desencadena y da pie a reflexionar sobre lo que podría haber ocurrido y ser imaginado como posibles alternativas a lo que está ocurriendo, definiéndose así la situación imaginada en la cual resulta clave confrontar lo que es real con lo que podría ser (Skovsmose \& Borba, 2004). Los procesos que nos ayudan a crear situaciones imaginarias, representando el complejo proceso de conceptualización en relación con que las cosas podrían hacerse de una manera diferente, es lo que se denomina como imaginación pedagógica (de esta manera se relacionan la SA y la SI).

Así, la situación dispuesta hace referencia a una alternativa práctica a la actual, teniendo en cuenta la situación imaginada. Dicha alternativa está constituida por actividades prácticas de planificación que resultan fundamentales y necesarias para establecer una situación que muestra cierta similitud con la situación imaginada. A tales actividades prácticas se les denomina organización práctica (de esta manera se relacionan la SA y la SD).

Por su parte, el razonamiento crítico hace referencia al proceso analítico de reconsiderar la situación imaginada a la luz de las experiencias relacionadas con la situación dispuesta. Constituyéndose, en tanto relación, como un proceso que considera (y condensa) la viabilidad tanto de la imaginación pedagógica como de los elementos innovadores de organización práctica.

De esta manera pensar en la investigación crítica implica entender que ella surge justamente de conceptualizar las posibilidades, circunstancia que le dan vida a la idea, cuestionar y problematizar la situación actual y considerar que lo que ocurre podría ser diferente. Para ello los datos (registrados en grabaciones de audio y video, entrevistas, escritos y producciones de los estudiantes) fueron analizados en Mancera (2020), considerando la noción de los actos dialógicos, circunstancia que es capitalizada en este artículo para responder la pregunta sobre qué caracteriza el conocer reflexivo de los Bernabé si 
consideramos, en tanto imaginación pedagógica, a la modelación matemática desde la perspectiva socio crítica.

\section{Fundamentaciones teóricas}

Dos aspectos que revisten gran importancia dentro de este ejercicio de conceptualización de posibilidades, en tanto imaginación pedagógica, son la modelación desde la perspectiva socio

crítica como un medio regular en el proceso educativo de los Bernabé Bernal y el conocer reflexivo.

Para ello, apoyado en las ideas de Skovsmose (1999) en relación con el hecho que para lograr un entendimiento mucho más específico del conocer reflexivo resulta determinante considerar la idea de modelación matemática, comienzo por ahondar entendimientos sobre la modelación para luego, a la luz de lo expresado por Skovsmose, referir a la idea del conocer reflexivo.

Teniendo como referente esta línea de desarrollo parto por señalar que, en el campo educativo colombiano, el desarrollo de la modelación matemática empezó a ser considerado, tal y como lo indica Villa (2009), a través de los Lineamientos Curriculares. Siendo reconocida como uno de los cinco procesos generales propuestos para ser desarrollados con los estudiantes. Al respecto, el documento de los Lineamientos considera:

Cuando hablamos de la actividad matemática en la escuela destacamos que el alumno aprende matemáticas "haciendo matemáticas", lo que supone como esencial la resolución de problemas de la vida diaria, lo que implica que desde el principio se integren al currículo una variedad de problemas relacionados con el contexto de los estudiantes.

La resolución de problemas en un amplio sentido se considera siempre en conexión con las aplicaciones y la modelación. La forma de describir ese juego o interrelación entre el mundo real y las matemáticas es la modelación. (Ministerio de Educación Nacional, 1998, p. 97)

Esta mirada de la modelación, como lo mencioné en Mancera (2020), podría considerarse cercana a concepciones sobre la alfabetización matemática que reflexionan sobre prácticas cotidianas recontextualizadas con las matemáticas académicas para centrar su atención en la capacidad de los estudiantes para usar las matemáticas que aprenden (o deben aprender) en la escuela. Es decir, encuentro que la idea de modelación descrita en los lineamientos se centra en una idea de alfabetización matemática para el desarrollo del capital humano (Jablonka, 2003). 
Mancera G. (2020). Imaginando cómo podría ser el conocer reflexivo de los Bernabé Bernal. Revista Latinoamericana de Etnomatemática, 13(4), 50-69. DOI: 10.22267/relatem.20134.71

Esta forma de concebir la modelación toma distancia de concepciones cuyos propósitos buscan, por ejemplo, reflexionar y evaluar la función de la matemática y de los modelos matemáticos en la sociedad (alfabetización matemática centrada en la ciudadanía), circunstancia que no es trivial si se tiene como propósito caracterizar el conocer reflexivo. Lo anterior, tal como lo expresé en Mancera (2020), me invita a tomar en consideración la noción de modelación desde la perspectiva socio crítica presentada por Araujo (2009) quien señala que:

La modelación matemática según EMC, de la forma como lo entiendo, reúne los aspectos presentados hasta ahora. Me preocupo con una formación política de los estudiantes, de tal forma que ellos actúen de forma crítica en nuestra sociedad en la que la presencia de las matemáticas es fuerte. Intento hacer del aula un espacio democrático, dialógico, preocupado por orientar a los estudiantes para que lleven estas actitudes para sus vidas en la sociedad.( p. 59)

De esta manera, como lo señala Valero (2012), las reflexiones que los estudiantes presentan, en escenarios de modelación, tienen en cuenta las implicaciones sociales y las consecuencias de tales reflexiones (y no solo de aspectos que involucran a las matemáticas). Lo anterior, trae consigo entendimientos sobre la subjetividad como los expresados por:

- García Oliveros et al. (2013) quienes consideran que la comprensión de los problemas de la enseñanza y el aprendizaje hoy en día pone en un primer plano asuntos como la subjetividad de los estudiantes y los procesos intersubjetivos.

- Torres (2000), quien señala que la subjetividad, por una parte, "Involucra un conjunto de normas, valores, creencias, lenguajes y formas de aprehender el mundo, conscientes e inconscientes, cognitivas, emocionales, volitivas y eróticas, desde los cuales los sujetos elaboran su experiencia existencial y sus sentidos de vida" (p. 4); y, por otra, en tanto se hacen presentes muchas voces cuando alguien se comunica, es intersubjetiva.

De lo dicho hasta aquí, reflexionar alrededor de la modelación, enmarcada en los principios de la Educación Matemática Crítica (EMC), implica tener en consideración una formación de ciudadanos críticos, en la que se promueva medios y formas que les permitan a los ciudadanos, y por supuesto a los Bernabé Bernal, considerar y comprender el papel que juegan las matemáticas en la sociedad, y en la vida misma, pues no poseer tales medios los ponen en una situación de desventaja. 
Consecuencia de lo anterior, resulta pertinente señalar que una educación basada en las ideas de la EMC debe viabilizar el desarrollo de una alfabetización matemática que permita a los ciudadanos ejercer una competencia democrática. Al respecto Skovsmose (1999) menciona:

La idea a la que he tratado de dar significado (y no demostrar) es: Si la alfabetización matemática tiene un papel que jugar en la educación - similar pero no idéntico al papel de la alfabetización - para tratar de desarrollar una competencia democrática, entonces la alfabetización matemática debe verse como una composición de diferentes competencias: la matemática, la tecnológica y la reflexiva. Y en especial: el conocer reflexivo tiene que desarrollarse para darle a la alfabetización matemática un carácter potenciador (pp. 129-130).

En resumen, la competencia de la EMC que se conecta con la competencia democrática general es el conocer reflexivo, que a su vez se relaciona con el matemático y el tecnológico, entendiendo tales conoceres como:

El conocer matemático, se refiere a la competencia normalmente entendida como habilidades matemáticas, incluyéndose las competencias en la reproducción de teoremas y pruebas, bien como al dominio de una variedad de algoritmos - esa competencia está enfocada en la educación matemática tradicional, y su importancia ha sido específicamente enfatizada por el movimiento estructuralista y por el movimiento de la matemática moderna- .

El conocer tecnológico, que se refiere a las habilidades en aplicar la matemática y a las competencias en la construcción de modelos. La importancia del conocer tecnológico ha sido enfatizada por la tendencia dirigida para aplicaciones en educación matemática, que afirma que, incluso si los estudiantes aprenden matemática, ninguna garantía existe de que la competencia desarrollada es suficiente cuando se trate de situaciones de aplicación. Esa competencia extra, es llamada competencia tecnológica.

El conocer reflexivo, que se refiere a la competencia de reflexionar sobre el uso de la matemática y evaluarlo. Las reflexiones tienen que ver con evaluaciones de las consecuencias del emprendimiento tecnológico (Skovsmose, 1999, pp. 115-116)

Según este autor, es claro que no se trata de identificar tres tipos de actividades que conduzcan a tres tipos de conocimiento. Al respecto, Skovsmose (1999) señala:

No podemos esperar encontrar ninguna relación lineal en el tiempo entre el desarrollo del conocer matemático, tecnológico y reflexivo. No se trata de que los niños primero tengan que adquirir una competencia matemática explícita antes de que sean capaces de usarla para resolver problemas tecnológicos. Tampoco podemos esperar que una competencia tecnológica tenga que desarrollarse antes de que puedan formarse una opinión razonada acerca de la solución propuesta (p. 129)

De esta manera, apoyado en Valero et al. (2015), pude asumirse que tal competencia coloca los conocimientos matemáticos en acción para cuestionar y, en consecuencia, hacer frente a la injusticia, circunstancia que cuestiona la idea de sólo saber matemáticas para tener un conjunto básico de conocimientos. Tratando de vislumbrar, de manera más concreta, estas ideas, a continuación presento, bajo la idea de ejemplaridad propuesta por Skovsmose (1999), 
Mancera G. (2020). Imaginando cómo podría ser el conocer reflexivo de los Bernabé Bernal. Revista Latinoamericana de Etnomatemática, 13(4), 50-69. DOI: 10.22267/relatem.20134.71

los desarrollos de uno de los grupos que participó en la investigación. Cabe apuntar que asumir a la ejemplaridad me ha implicado considerar como supuestos epistémicos: i) la idea de que en un fenómeno específico puede reflejarse una totalidad (objeto epistémico o de conocimiento); ii) es posible llegar a comprender la complejidad en su totalidad al concentrarse en un aspecto particular (la relación entre objeto de conocimiento y el sujeto) y iii) es posible para un sujeto dejarse atrapar, sorprender y absorber por una cuestión específica y, además, comprometerse por completo con el proceso de "llegar a conocer" (el sujeto epistémico).

\section{Los Cavaliers}

Como se señala en Camelo et al. (2017) el punto de partida en nuestras prácticas pedagógicas e investigativas debe emerger del conocimiento de nuestros estudiantes y de sus macro y micro contextos, por lo que preguntas del estilo: “¿quiénes son los infantes y jóvenes?, ¿cómo llegaron a ese contexto?, ¿qué momento histórico y social les correspondió vivir?, ¿cuáles son sus esperanzas y anhelos?” (p. 287) resultan ser relevantes.

En correspondencia con lo anterior, parto por señalar, como lo mencioné en Mancera (2020), que la comunidad de la localidad Rafael Uribe Uribe, en la que se encuentra ubicado el Colegio Bogotá $(\mathrm{CB})^{3}$, está expuesta a condiciones de vulnerabilidad social en el sentido mencionado por autores como Golovanevsky (2006). Es decir, en ella confluyen circunstancias que atentan, por ejemplo, contra: la capacidad de subsistencia de sus residentes, el acceso a mayores niveles de bienestar de sus habitantes y el ejercicio de sus derechos y deberes ciudadanos. Consecuencia de ello, en el entorno escolar se evidencian problemáticas como la inseguridad y la venta de estupefacientes tal y como es reportado por el periódico El Espectador (Redacción Bogotá, 2014).

Cabe señalar que aunque la pertenencia del profesor $\mathrm{Juan}^{4}$ al área de matemáticas podría implicar que su práctica pedagógica es orientada de acuerdo con el modelo constructivista

\footnotetext{
${ }^{3}$ Para conservar absoluta reserva de la identidad de la institución en la que se creó el ambiente de MM, me referiré a ella bajo el nombre ficticio de Colegio Bogotá (CB)

${ }^{4}$ Para conservar reserva de la identidad del profesor del área de matemáticas y de los estudiantes que participaron en el proyecto, haré referencia a ellos bajo nombres ficticios.
} 
(según se ha acordado en el área), él no es ajeno al desarrollo de prácticas pedagógicas de índole más crítico y social. Un ejemplo de ello es la actividad desarrollada en el año 2016 con un grupo de estudiantes, que posteriormente participaron en la producción de los datos del proyecto que se relaciona en el presente artículo, en la que indagaron sobre las ventajas y desventajas del acuerdo de paz entre el gobierno de Colombia y las Fuerzas Armadas Revolucionarias de Colombia (FARC).

Para desarrollar el ambiente de modelación desde una perspectiva socio crítica con el profesor Juan consideramos, como se mencionó en Mancera (2020), las ideas adelantadas en Salazar et al. (2017) en la que se plantean cinco etapas: i) escogencia del problema o tema a trabajar, que otorga gran importancia al macro y micro contexto; ii) desarrollo de una investigación exploratoria; iii) levantamiento de los datos y delineamiento de trayectorias de acción; iv) reinterpretación de la situación soportada en consideraciones matemáticas y desarrollo del problema; y v) análisis crítico de los desarrollos planteados ${ }^{5}$.

Como consecuencia, en la primera etapa, se les planteó inicialmente a los estudiantes que, distribuidos en grupos pensaran en problemáticas que fueran significativas para ellos de acuerdo con su micro y macro contexto y con sus propios intereses. Fue así como el profesor Juan, negoció y acordó con los estudiantes del curso $11-\mathrm{X}^{6}$ tan solo cuatro temáticas, de las nueve que surgieron inicialmente, para ser trabajadas durante el proyecto (colocando como presendente que un gran número de temáticas podría desbordar su trabajo en relación con la orientación).

En relación con el tipo de problemáticas que propusieron los estudiantes (en los siete grupos que se constituyeron: Brokling, Cavaliers, Dinamitas Girls, Divas, Magassa, Maldita sea y Warriors) debo señalar, como fue reportado en Mancera (2020), que:

- Tomaron distancia de aspectos como dificultades en la movilidad, infraestructuras inadecuadas, problemas de convivencia, hurto, tráfico y porte de estupefacientes, que podrían emerger de manera directa por el contexto en que se encuentran.

5 Estas cinco etapas han sido recontextualizadas a partir de consideraciones de la propuesta de Burak (1994, 2004).

${ }^{6}$ El grupo de estudiantes que participaron en el proyecto eran de grao undécimo. Para conservar reserva de la identidad de los participantes, de ser necesario me referiré al curso con el nombre ficticio 11-X. 
Mancera G. (2020). Imaginando cómo podría ser el conocer reflexivo de los Bernabé Bernal. Revista Latinoamericana de Etnomatemática, 13(4), 50-69. DOI: 10.22267/relatem.20134.71

- La experiencia sobre las implicaciones de votar a favor o en contra en el plebiscito sobre los acuerdos de paz en Colombia resultó significativa a la hora de proponer como temáticas en las que se centrarían sus ambientes de modelación a: la sobrepoblación mundial (grupos Cavaliers y Brokling), el embarazo en adolescentes (grupo Maldita Sea), la violencia intrafamiliar (grupos Divas y Magassa) y el cambio climático (grupos Warriors y Dinamita Girls).

Por efectos de espacio, en este documento centraré la atención en los desarrollos del grupo Cavaliers, quienes reflexionaron sobre el cambio climático a la hora de centrar como temática el cuidado de nuestros recursos naturales teniendo además una preocupación por el crecimiento de la densidad poblacional. Su motivación por esta temática radicó en que a principio del mes de febrero de 2017 Bogotá tuvo uno de los días con la mayor temperatura de su historia $\left(25^{\circ} \mathrm{C}\right)^{7}$ en contraste con $\operatorname{los} 2^{\circ} \mathrm{C}$ (a las 6.00 a.m.) dos semanas antes.

En relación con la etapa Desarrollo de una investigación exploratoria, resultó determinante la orientación del profesor Juan (y del profesor Manuel del área de español) apoyando la indagación y ampliación de los conocimientos, lo que les permitió acotar la problemática a modelar y plantear una pregunta (o al menos una idea orientadora de manera preliminar). De esta forma surgió en los Cavaliers, inicialmente, el cuestionamiento de si el agua potable existente en el mundo alcanzaría para todos dado el crecimiento poblacional.

En relación con la etapa Levantamiento de los datos y delineamiento de trayectorias de acción, los Cavaliers buscaron profundizar sus conocimientos sobre el tema, lo que les implicó buscar informaciones más precisas (en videos y documentos). De esta manera los Cavaliers consultaron y registraron en sus cuadernos sus hallazgos en relación con: i) qué es la tasa de natalidad, ii) qué es la tasa de mortalidad y iii) cómo se calcula tanto la tasa de natalidad como de mortalidad. Circunstancia que les permitió vislumbrar caminos para determinar cuántas personas nacen y cuántas mueren en un día, una semana, un mes y un año como se muestra en el siguiente fragmento de uno de los diálogos de los Cavaliers

\{C3-1\}: Brad Pitt. Pues esa yo me la sé [respondiendo cuando su compañera señala que deben buscar información sobre el tema].

\footnotetext{
${ }^{7}$ Hecho reportado en periódicos locales como El Espectador (Redacción Bogotá, 2017).
} 
Vea ... cada seis (6) segundos nace un bebé, ... cada once (11) muere una persona [esta información es suministrada de acuerdo con lo que encuentra, en ese preciso momento, en internet].

\{C3-2\}: Camila. ¿En serio?

\{C3-3\}: Brad Pitt. Es decir: por cada persona muerta... prácticamente hay dos (2) niños.

\{C3-4\}: Paula. Prácticamente, si...

\{C3-9\}: Johann. Hoy en día nacen 371.000 ... y mueren 201.000 personas también.

\{C3-10\}: Brad Pitt. Me perdí...

Pues podemos hacer... calcular cuántos al día. La calculadora. ¿cuántos al día?, ¿cuántos a la semana?, ¿cuántos al mes?, ¿cuántos al año?... y ¿hasta qué punto estaría sobrepoblado el mundo?... (Mancera, 2020, p. 164)

En relación con la etapa Reinterpretación de la situación soportada en consideraciones matemáticas y el desarrollo del problema, se evidencia la búsqueda por determinar el crecimiento poblacional, para lo que establecieron la diferencia entre las variables natalidad y mortalidad, circunstancia que les permitió ir soportando sus desarrollos con las matemáticas, como puede observarse en la siguiente ilustración (figura 1).

Figura 1. Imagen de los desarrollos de los Cavaliers

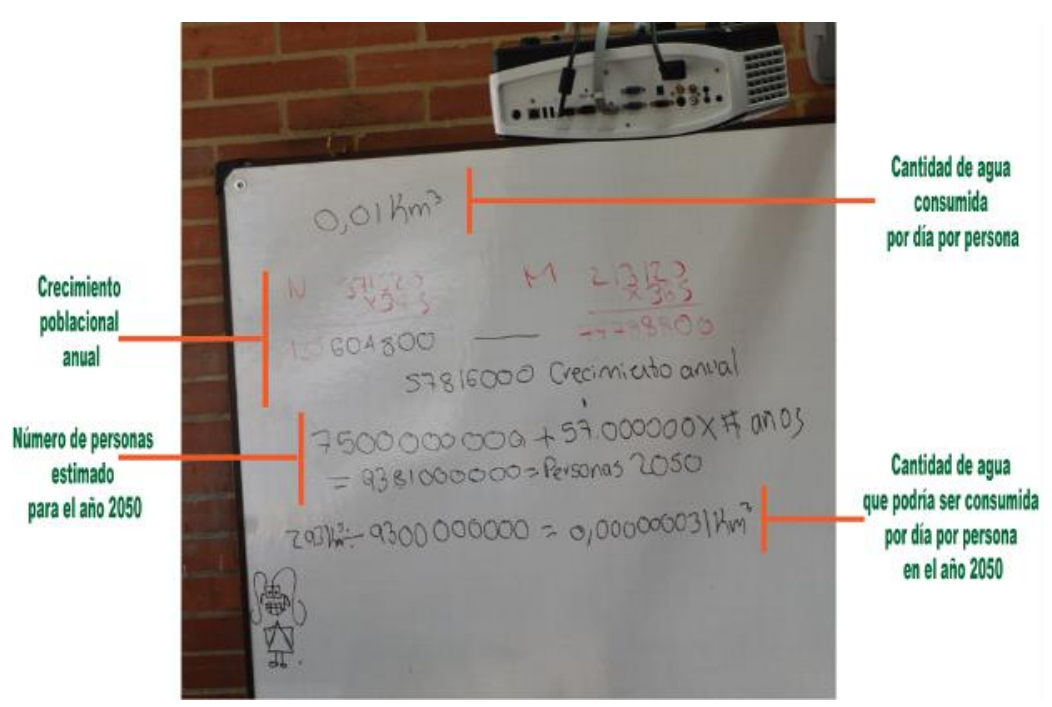

Fuente: Mancera (2020, p. 169)

En la que: 
Mancera G. (2020). Imaginando cómo podría ser el conocer reflexivo de los Bernabé Bernal. Revista Latinoamericana de Etnomatemática, 13(4), 50-69. DOI: 10.22267/relatem.20134.71

- El crecimiento poblacional anual es calculado como la diferencia entre el número de personas que nacen anualmente - natalidad-y el número de personas que mueren anualmente - mortalidad $-\left[135^{\prime} 604.800-77^{\prime} 788.800=57^{\prime} 816.000\right]$.

- El número de personas estimado para el año 2050 es calculado por la expresión:

$$
\begin{gathered}
7.500^{\prime} 000.000+57^{\prime} 000.000 \times \# \text { años }= \\
=9.381^{\prime} 000.000 \text { personas } 2050
\end{gathered}
$$

En la que 1) 7.500'000.000 corresponde al número estimado de personas en el mundo —en el momento de la toma de datos, según indagaciones hechas por internet-, 2) $57^{\prime} 000.000$ corresponde al número estimado del crecimiento poblacional anual —según el cálculo realizado por los Cavaliers-, y 3) la expresión “\# años” es considerada como la variable que representa el número de años a estimar la población — por ejemplo, para el año 2050 esta expresión fue reemplazada por los Cavaliers por $33-$.

- La cantidad de agua consumida por día por persona es asumida por la expresión $0,01 \mathrm{~km}^{3}$, según indagaciones hechas en internet por los Cavaliers. Cabe señalar que esta expresión fue cuestionada por el profesor Juan, como se verá más adelante.

- La cantidad de agua que podría ser consumida por día por persona en el año 2050 es estimada por la expresión:

$$
293 \mathrm{~km}^{3} \div 9.300^{\prime} 000.000=0,000000031 \mathrm{~km}^{3}
$$

En la que 1) $293 \mathrm{~km}^{3}$ corresponde a la cantidad de agua potable en el mundo - existente en el momento de la toma de datos según indagaciones hechas por el grupo en internet-, y 2) $9.300^{\prime} 000.000$ corresponde al número de personas estimado para el año 2050. (Mancera, 2020, p. 169)

Finalmente, respecto a la etapa Análisis crítico de los desarrollos planteados, debe señalarse que esta se desarrolló de manera transversal a la realización de cada una de las etapas, hecho que se vislumbra en los posicionamientos argumentados sobre sus propias problemáticas. Sin embargo, ante la inminente declaración del sindicato del Magisterio en relación con un paro nacional indefinido (lo que no permitía destinar más de una clase a la finalización del proyecto) el profesor Juan consideró pertinente realizar un debate, para que los grupos socializaran sus posiciones, reflexiones y conclusiones sobre la problemática desarrollada. En esta actividad, los Cavaliers, en términos generales, resaltan que:

- Obtener informaciones reales y confiables sobre las problemáticas desarrolladas les permitió mayor confiabilidad de sus propios procesos y reflexiones, de forma que vislumbraron el propósito de ir más allá de la realización de desarrollos y cálculos correctos y consistentes;

- No basarse en datos reales los pone en una posición en la que no pasarían del mundo de las especulaciones sobre las problemáticas, e inclusive desconocerían la propia 
existencia de ellas, pues los datos y los cálculos matemáticos les permitieron conocer los argumentos sobre sus problemáticas;

- Los desarrollos alcanzados y los cálculos matemáticos realizados influyeron en el pensamiento de los grupos sobre la problemática desarrollada, pues permitieron comprensiones, mayores claridades sobre los propios conceptos y argumentaciones y caminos para plantear soluciones a sus problemáticas. Esta circunstancia ayuda a centrar su atención sobre la manera en que nos podemos posicionar con respecto al uso de las matemáticas. (Mancera, 2020, p. 176)

\section{Consideraciones finales}

Tener como tela de fondo el conocer reflexivo de los Bernabé y los desarrollos de los estudiantes alrededor de un ambiente de modelación desde una perspectiva socio crítica, como el aquí presentado por el grupo Cavaliers, me permiten compartir con Skovsmose (1999) que tal conocer juega un papel importante en tanto que: i) permite indagar la manera en que la modelación matemática afecta (o no) el contexto social que dio origen al problema abordado, ii) tiene que captar la situación en la que las habilidades en la aplicación de las matemáticas y las competencias en la construcción del modelo se ponen en marcha y iii) considera que la base epistemológica de las reflexiones está en los aspectos sociológicos y éticos de la forma en que manejamos dicha tecnología.

Cabe señalar que si bien estos aspectos no son advertidos de manera puntual y determinante en los desarrollos de los Cavaliers; se logran vislumbrar (con intensidades diferentes) algunos trazos. A manera de ejemplo pongo en consideración los siguientes tres elementos:

En el primero, retomo señalamientos presentados en el debate. En este espacio, concretamente, el grupo manifiesta que no poder considerar en sus desarrollos datos reales los pone en un lugar en la que "no pasarían del mundo de las especulaciones sobre las problemáticas". Esta circunstancia permite vislumbrar entendimientos por parte de los Cavaliers sobre si la modelación matemática afecta (o no) el contexto social que dio origen al problema abordado. Especialmente cuando señalan que "inclusive desconocerían la propia existencia de ellas, las consecuencias" ponen de manifiesto que gracias a la modelación desde la perspectiva socio crítica pudieron problematizarse y significarse la problemática alrededor del cambio climático y el crecimiento poblacional. 
Mancera G. (2020). Imaginando cómo podría ser el conocer reflexivo de los Bernabé Bernal. Revista Latinoamericana de Etnomatemática, 13(4), 50-69. DOI: 10.22267/relatem.20134.71

Además, cuando señalan que "los datos y los cálculos matemáticos les permitieron conocer los argumentos sobre sus problemáticas" permiten localizar a las prácticas con las matemáticas como un rasgo importante.

En este sentido, entiendo que las prácticas con las matemáticas, consideran que los conocimientos curriculares pueden (y deben) ser utilizados en su vida cotidiana de tal manera que les permitan hacer frente a sus problemáticas. De esta manera:

las matemáticas escolares presentadas por los presupuestos de la MM deben permitirles utilizar aquellas matemáticas ya establecidas —inventadas por otros— para dar sentido y comprender situaciones de su propia realidad, de forma que den oportunidad para que ellos interpreten posibles significados que la matemática pueda tener. (Mancera, 2020, p. 209)

En el segundo elemento, apoyado en los desarrollos alcanzados por los Cavaliers, pueden observarse pensamientos que les permitieron mayor conciencia y sensibilidad sobre problemáticas sociales y ambientales como el cambio climático, el crecimiento poblacional y las consecuencias sobre la utilización indiscriminada de los recursos naturales, evento que pueden considerarse como consecuencia del hecho de captar la situación en la que las habilidades en la aplicación de las matemáticas y las competencias en la construcción del modelo se ponen en marcha. Particularmente reflexiones como "si seguimos así ... más personas, más urbanización, menos recursos" (señaladas en su infografía, figura 2) son consecuencia de los desarrollos alcanzados por medio del uso de las matemáticas.

Figura 2. Infografía de los Cavaliers
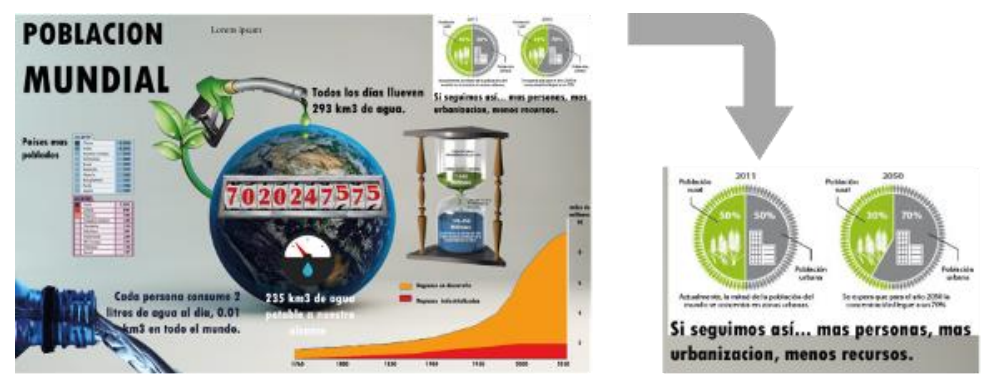

Fuente: Imagen adaptada de Mancera (2020, p. 175)

Cabe señalar, además, que esta toma de postura, junto con las profundidaes que de ella se derivaron, fue posible por la comprensión del lenguaje en general y del lenguaje matemático, de los estudiantes. Aspecto que evidencia, como otro rasgo central a la importancia de la lectura crítica y matemática. 
En consecuencia con lo anterior, leer crítica y matemáticamente, contempla el comprender, evaluar y analizar las diferentes prácticas que involucran las matemáticas. De esta manera, apoyado en Gutstein (2003), leer el mundo desde recursos matemáticos conlleva:

i) comprender las relaciones de poder, las inequidades de recursos y las disparidades de oportunidades entre diferentes grupos sociales; ii) entender la discriminación explícita basada en raza, clase social, género, lengua y otras diferencias; y iii) deconstruir los medios y otras formas de representación y usar las matemáticas para examinar. (Mancera, 2020, p. 208)

En el tercer elemento, coloco en consideración expresiones como: "Pero lo podemos mirar desde el punto de que: si hay sobrepoblación, alcanzará el alimento o no para suplir todas las necesidades de las personas" de las que se pueden derivar pensamientos que recaen, en tanto estructura humana, en la forma en que funcionamos. Lo que podría reflejar correspondencia con la idea de que la base epistemológica de las reflexiones está en los aspectos sociológicos y éticos de la forma en que manejamos dicha tecnología. En especial, líneas como \{C2-3\}, $\{\mathrm{C} 2-4\},\{\mathrm{C} 2-6\}$ del siguiente fragmento pueden reflejar correspondencia con la idea de que la base epistemológica de las reflexiones está en los aspectos sociológicos y éticos de la forma en que manejamos dicha tecnología.

\{C2-1\}: Camila. En esa sobrepoblación hay varios problemas, digamos que será que alcanzará el agua. Si no más así, ¿dónde es que es?, ¿en la Guajira? Ahí ya no hay agua [potable].

$\{\mathrm{C} 2-2\}$ : Paula. Por eso.

\{C2-3\}: Camila. Y los niños se están muriendo de sed, o algo así. No pueden ir a estudiar porque no se pueden bañar y eso...

$\cdots$

... pero ¿lo vamos a hacer en Colombia o en el mundo? [refiriéndose a si los alcances de su reflexión sobre la problemática serían "locales" —indagar tal situación en Colombia- o tendrían un carácter más abierto - que les permita tener una idea del comportamiento de tal problemática en el planeta-].

$\{$ C2-4\}: Brad Pitt y Paula. En el mundo.

$\{\mathrm{C} 2-5\}$ : Brad Pitt. In the world.

\{C2-6\}: Paula. ¿Alcanzará el agua para todos?, ¿la comida? ... (Mancera, 2020, p. 161)

Además, advierten pensamientos sobre una ética hacia el otro que sufre, derivandose, como rasgos determinantes a la colectividad y el pensar en el otro.

Al respecto, entiendo que pensar en el otro, implica diferenciar el rostro de la cara. Ello, por ejemplo, compromete, en términos de Mèlich (2014) a no ser indiferentes frente al dolor del otro (“¿Alcanzará el agua para todos?, ¿la comida?). De esta manera para Mèlich, basado en 
Mancera G. (2020). Imaginando cómo podría ser el conocer reflexivo de los Bernabé Bernal. Revista Latinoamericana de Etnomatemática, 13(4), 50-69. DOI: 10.22267/relatem.20134.71

los planteamientos de Lévinas, centrarnos en el rostro y no en la cara (el color de la piel, el género, los rasgos físicos ...) se constituye en una ética como filosofía primera que se basa en:

la sensibilidad al mal, al dolor y al sufrimiento (de la no indiferencia hacia el dolor del otro, reevaluando la idea ontológica del yo soy yo y tú eres tú). Así, la alternativa ante la diferencia entre el yo y el tú basado en la in-diferencia es no pensarnos como caras sino como rostros. (Mancera, 2020, p. 208)

En relación con la colectividad, de la manera en que la consivo, tiene el propósito de "pensar y actuar siendo conscientes de que son parte de una sociedad, en la que cada uno es "un serentre-los-otros". En términos arendtianos, no debemos perder nuestra condición humana de ser una subjetividad diferente entre la pluralidad" (Mancera, 2020, p. 209).

De esta manera, en tanto ejercicio analítico, retomar la premisa de reconsiderar la situación imaginada a la luz de los cuatro rasgos relacionados (pensar en el otro, leer crítica y matemáticamente, prácticas con las matemáticas y la colectividad) como características del conocer reflexivo, me ha implicado reflexionar sobre las situaciones imaginadas a través de los desarrollos de la situación dispuesta (condensados para este documento en los desarrollos de los Cavaliers), teniendo como propósito una posible respuesta a la pregunta "¿Qué caracterizaría el conocer reflexivo de los Bernabé Bernal si en su historia escolar la modelación matemática desde la perspectiva socio crítica resultara ser un medio regular en su formación?"

Esta línea de consideraciones y reflexiones me han permitido imaginar relatos diferentes al presentado al inicio del presente capítulo sobre los Bernabé Bernal, uno de ellos, a manera de ejemplo, podría ser el que presento a continuación:

La novela narra las circunstancias de vida de un hombre vulnerado socialmente que ha recibido una formación basada en la modelación matemática desde la perspectiva socio crítica, circunstancias que lo alejan de ser ingenuo y tímido; por el contrario, Bernabé sigue siendo un ser inteligente que a pesar de estas circunstancias de vida es un ser empoderado que busca liberarse de cualquier clase de subordinación o dependencia gracias a una idea escolar que se fundamenta en una alfabetización matemática que hace referencia a la evaluación crítica de aspectos de su cultura, la cual está — claramente - impregnada de prácticas con las matemáticas, logrando, además, aprendizajes por sí mismo de manera reflexiva (autodidacta). Bernabé no se concibe como un ser que pueda ser explotado por sus compañeros y futuros jefes y sus superiores para lograr sus aspiraciones sociales, políticas y económicas ni como un ser que busque explotar a los demás, pues su mirada se basa en no ser indiferentes frente al dolor del otro. En este relato, imagino a Bernabé Bernal, además, como un ser culto y honesto que comprende, evalúa y analiza las diferentes prácticas que involucran las matemáticas gracias a 
su lectura crítica y matemática; que actúa siendo consciente que es parte de una sociedad, en la que cada uno es "un ser-entre-los-otros" lo que le permite constituirse en un ser que asume la colectividad. Un ser que - a pesar de encontrarse en un círculo de escasez, de falta de oportunidades, de la lucha diaria por su sustento y el de su familia - busca permanentemente liberarse de cualquier clase de subordinación o dependencia. Bernabé, a pesar de sus circunstancias, no traiciona sus principios éticos, teniendo como gran satisfacción el deber cumplido y el orgullo de tener la esperanza de un mejor mañana, que nos proporcione mejores oportunidades para todos. (Mancera, 2020, pp. 210-211)

\section{Bibliografía}

Araújo, J. (2009). Uma Abordagem Sócio-Crítica da Modelagem Matemática: A perspectiva da educação matemática crítica. ALEXANDRIA Revista de Educaçãoem Ciência e Tecnologia, 2(2), 55-68.

Barbosa, J. C. (2006). Mathematical modelling in classroom: A socio-critical and discursive perspective. ZDM, 38, 293-301.

Camelo, F. J., Mancera, G., \& Salazar, C. (2017). Una mirada a la equidad en nuestras prácticas desde la dimensión política de la educación matemática. Revista Colombiana de Educación, 73, 283-300.

García, N. (2002). Latinoamericanos buscando lugar en este siglo (1. ed). Paidós.

García Oliveros, G., Valero Dueñas, P. X., Salazar Amaya, C., Mancera Ortiz, G., Carmelo Bustos, F. J., \& Romero Rey, J. H. (Eds.). (2013). Procesos de inclusión / exclusión: Subjetividades en educación matemática (Primera edición). Universidad Pedagógica Nacional : Aalborg University, Denmark: Universidad Distrital Francisco José de Caldas: República de Colombia, COLCIENCIAS, Departamento Administrativo de Ciencia, Tecnología e Innovación.

Golovanevsky, L. (2006). Vulnerabilidad y transmisión intergeneracional de la pobreza. Un abordaje cuantitativo para la Argentina en el siglo XXI. [Universidad de Buenos Aires. Facultad de Ciencias Económicas]. http://bibliotecadigital.econ.uba.ar/download/tesis/1501 1179_GolovanevskyL.pdf

Guba, E. G., Lincoln, Y. S., \& others. (1994). Competing paradigms in qualitative research. Handbook of qualitative research, 2(163-194). http://www.gdufs.biz/10guba_lincoln_94.pdf

Gutstein, E. (2003). Teaching and Learning Mathematics for Social Justice in an Urban, Latino School. Journal for Research in Mathematics Education, 34(1), 37. https://doi.org/10.2307/30034699

Hein, N., \& Biembengut, M. (2006). Modelaje matemático como método de investigación en clases de matemáticas. M. Murillo (presidente), Memorias del $V$ festival internacional de matemática, 1-25.

Jablonka. (2003). Mathematical Literacy. En Bishop, Clements, Keitel, Kilpatrick, \& Leung (Eds.), Second International Handbook of Mathematics Education (pp. 75-102). Kluwer Academic Publishers.

Mancera, G. (2020). Conocer reflexivo en contextos de modelación matemática desde una perspectiva socio crítica. Universidad Federal de Minas Gerais.

Mèlich, J. (2014, diciembre 4). Pensando la Educación desde Emmanuel Levinas: Vol. Educación 
Mancera G. (2020). Imaginando cómo podría ser el conocer reflexivo de los Bernabé Bernal. Revista Latinoamericana de Etnomatemática, 13(4), 50-69. DOI: 10.22267/relatem.20134.71

[Vídeo]. Facultad de Educación - Universidad de Antioquia. https://www.youtube.com/watch?v=DXZipK7yaas

Ministerio de Educación Nacional. (1998). Lineamientos curriculares. Área de matemáticas. Cooperativa editorial Magisterio.

Redacción Bogotá. (2014, septiembre 4). Distrito, preocupado por inseguridad en Rafael Uribe Uribe. ELESPECTADOR.COM.

Salazar, Mancera, \& Camelo. (2017). Una propuesta para el desarrollo de prácticas pedagógicas de modelación matemática en la perspectiva socio crítica. Memorias EDEM 4. Encuentro Distrital de Educación Matemática EDEM-4 "Cultura, sociedad y escuela en la educación matemática del Distrito capital”, Bogotá - Colombia.

Skovsmose. (1999). Hacia una filosofía de la educación matemática crítica (Valero, Trad.; Primera). una empresa decente.

Skovsmose. (2015). Pesquisando o que não é, mas poderia ser. En C. Lopes \& D’Ambrosio, Vertentes da Suversão na Producção Científica em Educação Matemática (pp. 63-90). Mercado das Letras.

Skovsmose, \& Borba, M. (2004). Research methodology and critical mathematics education. En Researching the socio-political dimensions of mathematics education (pp. 207-226). Springer. http://link.springer.com/content/pdf/10.1007/1-4020-7914-1_17.pdf

Torres, A. (2000). Educación popular, subjetividad y sujetos sociales. Pedagogía y Saberes, 15, 5. https://doi.org/10.17227/01212494.15pys5.14

Valero, P. (2012). La educación matemática como una red de prácticas sociales. En Educación matemática crítica: Una visión sociopolítica del aprendizaje y la enseñanza de las matemáticas. (Valero, P.; Skovsmose, O., pp. 299-326). Ediciones Uniandes.

Valero, P., Andrade, M., \& Montecino, A. (Eds.). (2015). Lo político en la educación matemática: De la educación matemática crítica a la política cultural de la educación matemática. Revista Latinoamericana de Investigación en Matemática Educativa, 18(3), 287-300. https://doi.org/10.12802/relime.13.1830

Villa, J. (2009). Presente y futuro de la investigación en modelación en Educación Matemática en Colombia. Memorias $10^{\circ}$ Encuentro Colombiano de Matemática Educativa, 8. https://www.researchgate.net/publication/277846514_Presente_y_futuro_de_la_investigaci on_en_modelacion_en_Educacion_Matematica_en_Colombia

Vithal, R. (2000). Re-Searching Mathematics Education from a Critical Perspective. Papers Bienial International Conference on Mathematics Education and Society. 2nd Bienial International Conference on Mathematics Education and Society, Montechoro - Portugal. 